\title{
Avaliação de desempenho na organização de gestão do trabalho no município de São Tomé-RN
}

RESUMO | Objetivo: refletir sobre a necessidade de implantação da avaliação de desempenho dos profissionais de saúde do município de São Tomé-RN. Método: Trata-se de um ensaio reflexivo, de caráter descritivo e de abordagem qualitativa. Resultados: Tem-se a necessidade de sensibilizar a gestão, na perspectiva de implantar uma avaliação de desempenho, a partir de instrumentos, tendo em vista várias necessidades tais como: perfil profissional, assiduidade, comportamento profissional, capacitações de educação continuada, viabilizar desempenho eficaz do profissional, melhorar condições de trabalho e qualidade de vida no trabalho. Conclusão: Para concretizar a implantação de avaliação de desempenho é preciso que haja um bom planejamento; realizar diagnósticos na gestão com bases nos problemas levantados, constatar problemas não é o suficiente para provocar mudanças necessárias. Faz-se imperativo, portanto, empenhar-se na construção de estratégias que possam promover mudanças no cotidiano dos serviços de saúde que possam vir a contribuir para melhoria da gestão.

Palavras-chaves: Avaliação de desempenho; Educação em saúde; Gestão em saúde.

\begin{abstract}
Objective: to reflect on the need to implement the performance evaluation of health professionals in the municipality of São Tomé-RN. Method: This is a reflective essay, with a descriptive character and a qualitative approach. Results: There is a need to sensitize management, with a view to implementing a performance evaluation, based on instruments, in view of various needs such as: professional profile, attendance, professional behavior, continuing education skills, enabling effective performance professional, improve working conditions and quality of life at work. Conclusion: In order to implement the performance evaluation, there must be good planning; making diagnoses in management based on the problems raised, finding problems is not enough to cause necessary changes. It is imperative, therefore, to commit to the construction of strategies that can promote changes in the daily life of health services that may contribute to improve management.
\end{abstract}

Keywords: Performance evaluation; Health education; Health management.

RESUMEN | Objetivo: reflexionar sobre la necesidad de implementar la evaluación del desempeño de los profesionales de la salud en el municipio de São Tomé-RN. Método: Se trata de un ensayo reflexivo, con carácter descriptivo y enfoque cualitativo. Resultados: Existe la necesidad de sensibilizar a la gestión, con miras a implementar una evaluación de desempeño, basada en instrumentos, ante diversas necesidades tales como: perfil profesional, asistencia, comportamiento profesional, capacidades de educación continua, posibilitando un desempeño efectivo profesional, mejorar las condiciones laborales y la calidad de vida en el trabajo. Conclusión: Para implementar la evaluación de desempeño, debe haber una buena planificación; haciendo diagnósticos en la gestión basados en los problemas planteados, encontrar problemas no es suficiente para provocar los cambios necesarios. Por tanto, es imperativo apostar por la construcción de estrategias que puedan promover cambios en la vida diaria de los servicios de salud que contribuyan a mejorar la gestión.

Palabras claves: Evaluación del desempeño; Educación para la salud; Manejo de la salud.

\section{Aldenira Joacla Caetano da Silva}

Enfermeira. Universidade Federal do Rio Grande do Norte. Especialista em Ginecologia e Obstetrícia, Especialista em Urgência e Emergência, Especialista em Auditoria nos Serviços de Saúde.

ORCID: 0000-0002-4815-2930

\section{Emanoele Belchior de Medeiros}

Enfermeira. Universidade Federal do Rio Grande do Norte. Especialista em Ginecologia e Obstetrícia, Especialista em Urgência e Emergência.

ORCID: 0000-0002-2625-6798

\section{Illana Cecylia Silva Basílio}

Enfermeira, Centro Universitário Facex (UNIFACEX).

ORCID: 0000-0002-0000-4170

Recebido em: 23/11/2020

Aprovado em: 01/12/2020

\section{Janaina Kelly Alves Barbosa}

Enfermeira. Universidade Federal do Rio Grande do Norte. Especialista em Ginecologia e Obstetrícia, Especialista em Urgência e Emergência, Especialista em Auditoria nos Serviços de Saúde.

ORCID: 0000-0002-4815-2930

\section{Robson Egidio da Silva}

Enfermeiro. Faculdade Mauricio de Nassau. Especialista em UTI.

ORCID: 0000-0003-3665-0761

INTRODUÇÃO

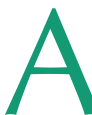
avaliação é uma atividade que acompanha a própria história do homem, tida como inerente ao próprio processo de aprendizagem, e na atualidade assume cada vez mais um caráter polissêmico em sua conceituação, abrigando múltiplas realidades e múltiplos referenciais de análise.

As práticas de avaliação de desempenhos não são novas. Desde que o homem deu emprego ao outro seu trabalho passou a ser avaliado. ${ }^{1} \mathrm{~A}$ avaliação é uma atividade que acompanha a própria história do homem, tida como inerente ao processo de aprendizagem. ${ }^{2}$

Partindo dos princípios da Política Nacional de Humanização (PNH), enfatiza-se a participação dos diferentes sujeitos, gestores, trabalhadores, usuários, pesquisadores, apoiadores, nos processos avaliativos. Esses são essenciais em todas as fases dos desenhos avaliativos: identificação de situações a serem avaliadas, conceitualização, demarcação de objetivos, critérios de re- 
presentação/indicadores, mensuração, interpretação e validação dos dados. ${ }^{3}$

Para se caracterizar como método e dispositivo da Política de Humanização, a avaliação reveste-se com os princípios mais centrais dessa Política, dentre eles a compreensão de que as instituições de saúde têm como finalidades primordiais alcançar a produção/prestação de serviços e também a produção de sujeitos. ${ }^{4} \mathrm{Na}$ mira do seu planejamento e avaliação (dos serviços/ instituições), deve-se portanto recortar indicadores relacionados tanto às dimensões de saúde e satisfação dos usuários, quanto aos movimentos institucionais, e indicativos do desenvolvimento e crescimento profissional e pessoal dos trabalhadores/equipes. Em suma, avaliação que possibilite mudanças nas situações/serviços, e capacitação e desenvolvimento dos sujeitos envolvidos.

É esse contexto de complexidade da Humanização que deve abrigar e reorientar o uso da informação e os processos de monitoramento e avaliação. Sobretudo deve-se partir do princípio de que são as próprias mudanças desejadas nos modelos de atenção e de gestão que devem orientar mudanças na forma de se lidar com a informação e avaliação. As ações de promoção da saúde apontam desafios e perspectivas teóricos metodológicas de abordagens nessa área, o que torna um desafio no campo de programas de políticas públicas; portanto vê-se a necessidade cada vez mais da intervenção de uma avaliação de desempenho,uma vez que permite maior aproximação da realidade. ${ }^{5}$

A avaliação em saúde, de caráter institucional permite conhecer o desempenho do setor e aperfeiçoar os resultados de acordo com o contexto em que a instituição se insere, corrigindo os problemas detectados e possibilitando a conquista de novas metas. ${ }^{6}$

É nesse contexto que se insere o presente artigo que objetiva refletir sobre a necessidade de implantação da avaliação de desempenho dos profissionais de saúde do município de São Tomé-RN.

\section{MÉTODO}

Trata-se de um ensaio reflexivo, de caráter descritivo e de abordagem qualitativa, que propõe uma discussão acerca necessidade de implantação da avaliação de desempenho dos profissionais de saúde do município de São Tomé-RN.

Para a busca e análise do referencial estudado foi realizada uma revisão narrativa da literatura, utilizando-se as seguintes palavras-chave: Avaliação de desempenho, Educação em saúde E Gestão em saúde.

Além da reflexão, propõe-se uma metodologia de avaliação de serviços de saúde, entendendo-a como um processo permanente e sistemático, articulada às ações implementadas, com vistas a subsidiar a definição de problemas, mensurar impactos das ações e reorientar as práticas sanitárias.

\section{RESULTADOS}

O município de São Tomé (RN) é o décimo maior em extensão no Estado do RN estadual, e sua população atual segundo o Instituto Brasileiro de Geografia e Estatística (IBGE) é de 11.501 habitantes dividindo-se em 51,90\% residentes na zona urbana e $48,10 \%$ residente na zona rural, apresentando um índice de desenvolvimento 0,613 . Tendo como principais atividades econômicas a agropecuária, extrativismo e o comércio.

O município dispõe em torno de cento e cinqüenta profissionais de saúde efetivos e contratados, compõe-se em sua estrutura organizacional de 08 (oito) unidades de saúde, um hospital de pequeno porte, onde numero de leitos existentes para suprir as necessidades emergenciais e de urgência através do Sistema Único de Saúde são 32 (trinta e dois) leitos, com profissionais em plantão 24 horas.Para tanto destina-se para essas unidades 05 equipes do Programa Saúde da família , sendo 02 na zona rural e 03 na zona urbana. O município dispõe de um núcleo de Apoio ao Atendimento da Família (NASF), além de contar com o programa mais médicos.

A avaliação de serviços de saúde possui obstáculos, tanto conceituais quanto operacionais, que vão da complexidade do objeto, da dificuldade na sistematização de ferramentas que possam contribuir na sua apreensão, à obtenção dos dados, fontes, documentos e informações confiáveis.

Sugere-se a utilização de um instrumento na perspectiva de estabelecer prioridades, que seja participativo no sistema em si, contribuindo decisivamente para ressaltar os aspectos políticos e interpessoais da avaliação de desempenho. Serão utilizados os seguintes marcadores para avaliação: 1) Determinantes da Saúde (Ambientais, Socioeconômicos e Demográficos); 2) Condições de Saúde da População (Mortalidade, Morbidade); 3) Sistema de Saúde (Recursos, Estrutura); 4) Desempenho dos Serviços de Saúde (Eficiência, Eficácia, Efetividade, Acesso).

Um modelo de avaliação de desempenho para ser bem sucedida, deve respeitar as características culturais da organização que o adota. ${ }^{7}$ Idealmente um modelo de avaliação de desempenho deve trazer benefícios para todos os seus usuários.

Possíveis fragilidades encontradas: 1) não sensibilização da gestão do sistema; 2) ausência de informação da secretaria municipal de saúde; 3) Qualidade do serviço; 4) Vínculo empregatício precários; 5) Rotatividade dos profissionais da saúde.

Quanto ao processo avaliativo, tem-se que o mesmo deverá ser realizado pela equipe responsável, assim como é da gestão de saúde a responsabilidade de aplicar a avaliação de desempenho. Será necessário o fornecimento de informações, visto que a avaliação de desempenho e feedback são um jogo de percepções e precisa ser uma ferramenta de gestão e de gestores. O processo de avaliação, discutido por diversos autores, envolve, necessariamente, um julgamento, uma atribuição de um valor, uma medida de aprovação ou desaprovação a uma política ou programa. A avaliação é um instrumento que irá contribuir para melhorar o desempenho dos programas sociais, tornando-se uma instância de aprendizagem que permite reelaborar e melhorar esses programas.

\section{DISCUSSÃO}

A busca da qualidade da atenção dos serviços de saúde deixou de ser uma atitu- 
de isolada e tornou-se hoje um imperativo técnico e social. A sociedade está exigindo cada vez mais a qualidade dos serviços a ela prestados, principalmente por órgãos públicos. Esta exigência torna fundamental a criação de normas e mecanismos de avaliação e controle da qualidade assistencial. A avaliação é, em especial, parte fundamental no planejamento e na gestão do sistema de saúde. Um sistema de avaliação efetivo deve reordenar a execução das ações e serviços, redimensionando-os deforma a contemplar as necessidades de seu público, dando maior racionalidade ao uso dos recursos.

Autores $^{8}$ referem que as novas responsabilidades com a gestão do sistema de saúde requerem a incorporação da avaliação como componente do processo de planejamento, como atividade capaz de subsidiar as tomadas de decisões e como elemento auxiliar nas iniciativas voltadas para a mudança do modelo assistencial. Sua adoção como uma atividade sistemática ao interior da gestão municipal, e completamos estadual e federal, pode ser considerado um dos indicadores de modificação do modelo assistencial.

Desse modo, avaliar é uma responsabilidade e as instituições não podem se furtar em exercê-la. Institucionalizar a avaliação significa incorporá-la ao sistema, possibilitando monitorar a capacidade dos serviços em responder às necessidades em saúde; acompanhar os efeitos das intervenções; identificar e corrigir problemas; enfim, retroalimentar equipes de saúde, gestores, políticos e comunidades. ${ }^{9}$

Neste sentido, para que este processo se institua de forma efetiva, mecanismos pactuados de monitoramento e avaliação da gestão do SUS devem ser implantados em todas as unidades federadas, estabelecendo-se a responsabilização dos estados e municípios e do gestor federal no âmbito do SUS, com vistas ao fortalecimento da capacidade de gestão pública da saúde.

No âmbito da gestão, fica patente que as práticas de monitoramento e avaliação constituem-se num carreador de responsabilidades, e de ações rumo a objetivos definidos previamente, onde devem ser previstas decisões em meio a situações diversas e impostas por circunstâncias que os gestores e as equipes que os assessoram muitas vezes não escolhem, sendo necessário a instituição de mecanismos e estratégias que potencializem e consolidem a sua institucionalização.

Além disso, tem-se que o monitoramento e a avaliação são ferramentas críticas para a gestão. ${ }^{10} \mathrm{O}$ monitoramento é fundamental para o acompanhamento rotineiro de informações prioritárias, tanto para o processo de implementação de um programa, isto é, para o acompanhamento de seu desempenho. A avaliação por sua vez traz a visão de julgamento, prática de intervenções que auxiliam na tomada de decisão, ação capaz de subsidiar mudanças na construção e/ou na implementação de programas, projetos ou políticas de saúde.

\section{CONCLUSÃO}

A avaliação é conceituada, como emissão de juízo de valor sobre um projeto por meios de parâmetros definidos. Para concretizar a implantação de avaliação de desem- penho é preciso que haja um bom planejamento, realizar diagnósticos na gestão com bases nos problemas levantados.

Constatar problemas não é suficiente para provocar mudanças necessárias, faz-se imperativo, portanto empenhar-se na construção de estratégias que possam promover mudanças no cotidiano dos serviços de saúde que possam vir a contribuir para melhoria da gestão como: montar planos de ações com indicadores e identificadores, capacitar as equipes de saúde através de programas de educação permanente. Isso pode ser realizado pela adoção de novas práticas, habilidades, capacidade e competências gerenciais e técnicas de relacionamento interpessoal.

Independentemente do mecanismo de avaliação do desempenho que as organizações prestadoras de serviços de saúde apliquem, a sua utilização deverá ser compreendida não apenas como uma necessidade imposta pelas atuais exigências do sector mas sim como uma realidade implícita na nova cultura de avaliação promotora da melhoria contínua da qualidade na prestação de serviços de saúde.

Será importante destacar que os indicadores quando utilizados de forma adequada deverão ser apenas vistos como "marcadores" que possibilitem a identificação de problemas e não como elementos utilizados no julgamento dos prestadores de serviços de saúde. Além disso, as variações dos dados deverão ser analisadas de forma muito cuidadosa, analisando as causas, pois podem refletir variações na qualidade da prestação ou variações na qualidade dos dados.

\section{Referências}

1. Cardoso MS. Avaliação da influência da Gestão do Desempenho nos Funcionários do Hospital de Clínicas de Porto Alegre [monografia]. Porto Alegre (RS): Universidade do Estado do Rio Grande do Sul; 2005.

2. Deslandes SF, Assis SG. Abordagem quantitativa e qualitativa em saúde: 0 diálogo das diferenças. In: Minayo MCS, Deslandes SF. Caminhos e pensamentos: epistemologia e métodos. Rio de Janeiro. Fiocruz; 2002. p.195-223.

3. Hart ZMA. Avaliação em saúde: dos modelos conceituais à prática na análise da implantação de programas. Rio de Janeiro (R): Fiocruz; 1997.

4. Campos GWS. Um método para análise e co-gestão de coletivos. São Paulo (SP): Hucitec; 2000.

5. Pontes BR. Planejamento, recrutamento e seleção de pessoal. São Paulo: LTR; 2001.
6. Nickel DA, Calvo MCM, Caetano JC. Modelo de avaliação da atenção em saúde bucal. Pesqui Bras Odontopediatria Clín Integr. 2009; 9:373-9.

7. Oliveira-Castro GA. Avaliação de Desempenho em Psicologia: Questões conceituais e metodológicas. Psicologia: Teoria e Pesquisa. 1994; 10(3): 355-374.

8. Brito TA, Jesus CS. Avaliação de serviços de saúde: aspectos conceituais e metodológicos. Revista Digital. 2009; 139

9. Takeda S, Yves T.Avaliar, uma responsabilidade. Cien Saude Colet. 2006; 11(3):569-571 10. Santos EM, Cruz MM, Decotelli PV, Chispin PPM, Abreu DMF. Situ(ação): reflexões e desafios sobre a institucionalização da prática avaliativa no PN DST/Aids. In: Onocko RC Furtado JF. Desafios da avaliação de programas e senviços em saúde. Campinas: Editora Unicamp; 2011. p. 205-222. 